\title{
A interface entre Saúde Pública e Cibercultura
}

\author{
The interface between public health and cyberculture
}

Eduardo Jorge Sant Ana Honorato ${ }^{1}$

${ }^{1}$ Instituto Fernandes Figueira, Fiocruz._Av. Rui Barbosa 716, Flamengo. 22.250-020 Rio de Janeiro RJ Brasil. eduhonorato@ hotmail.com

\begin{abstract}
This is an opinion piece that proposes a reflection on the current status of the interface between cyberculture and public health and its use as a means for research, not as a mere tool. Cyberculture thus represents a new form of interface between people. And it is precisely "through" and "by means of" social relations that individuals acquire skills and communication techniques. The forms and the means of the relationship alters, but the ends remain unchanged, namely to be in contact with other humans. In recent decades, with the advent of computers, the Internet and all the technological apparatus, human relationships are dependent on them, which is the modern so-called cyberculture. This now affects all areas of activity, and public health cannot be left behind, taking advantage of it and its benefits for its development. It is necessary to keep abreast of these changes and raise them from the theoretical to the practical plane, not only implementing public health policies but also taking the sociovirtual aspects into consideration. It is also necessary for the professionals involved to be updated on new forms of communication, interaction, research methodology, preparation of instruments, sampling approaches and all other phenomena arising from cyberculture that will work in partnership with public health.
\end{abstract}

Key words Cyiberculture, Public health, Research
Resumo Este artigo, de caráter opinativo, propõe uma reflexão sobre como está a relação entre a cibercultura e a saúde pública e sua utilização como meio de pesquisa, e não apenas como instrumento. Cibercultura seria então uma nova forma de relação entre sujeitos. É justamente "através" e "pelas" relações sociais que os indivíduos adquirem habilidades técnicas e comunicação. Mudam-se as formas relacionais, os meios, mas os fins permanecem os mesmos: o de estarem em contato com outros humanos. Nas últimas décadas, com o advento do computador, Internet e todo aparato tecnológico, as relações humanas se veem intermediadas por estes, o que caracteriza algo atual, denominado de cibercultura. Esta hoje influencia todas as áreas de atuação e a saúde pública não pode ficar de fora, entendendo esta e seus benefícios para seu desenvolvimento. Precisamos estar atentos a essas mudanças e trazê-las do plano teórico para o plano prático, implementando não somente políticas públicas de saúde que levem em conta o sócio-virtual, mas também, enquanto profissionais, nos atualizarmos sobre as novas formas de comunicação, interação, metodologia de pesquisa, elaboração de instrumentos, abordagens de amostragem e todos os demais fenômenos decorrentes da cibercultura que trabalharão em parceria com a saúde pública.

Palavras-chave Cibercultura, Saúde pública, Pesquisa 


\section{Introdução}

Os dados do Suplemento da Pesquisa Nacional por Amostra de Domicílios ${ }^{1}$ afirmam que " 56 milhões de pessoas de dez anos ou mais de idade acessaram a Internet pelo menos uma vez". Antes eram jovens estudantes, hoje se tem uma distribuição mais homogênea entre classes sociais, faixas etárias e ocupações. $86 \%$ dos entrevistados afirmam que o principal objetivo em utilizar a Internet é para comunicação com outras pessoas, o que faz dessa interação ponto central da Rede ${ }^{1}$.

As questões mais urgentes em saúde pública se fazem presentes no entendimento desse novo espaço no qual a sociedade atual está inserida. Deixamos de ser seres sociais para sermos seres sócio-virtuais. Assim como tivemos que transdisciplinar na saúde pública ao longo da história, em diversas áreas, como epidemiologia, bioestatística, humanas, sociais etc., hoje precisamos ultrapassar esse bloqueio e termos uma saúde pública que entenda as tecnologias de informação e os sujeitos que nela interagem e que estes são os nossos usuários e foco de trabalho e pesquisas. Não podemos negar a importância que as tecnologias assumiram no cotidiano social, $\mathrm{e}$ deixar esse fenômeno para trás seria atrasar nossos conhecimentos sobre a realidade social na qual a saúde pública deve intervir. As tecnologias de informação deixaram ser algo exclusivo de classes sociais mais favorecidas para adentrarem no dia a dia de todos os sujeitos da atualidade. O artigo, de caráter opinativo, propõe uma reflexão sobre como está a relação entre a cibercultura e a saúde pública e sua utilização como meio de pesquisa, e não apenas como instrumento.

\section{Saúde Pública e Cibercultura}

Como se posiciona Le Breton ${ }^{2}$, o corpo, que transcende o físico, se perde dentro de si mesmo, num labirinto em que ele próprio definirá as direções em que deverá seguir. O mundo virtual é compreendido como parte do real ${ }^{2}$, porém este ciberespaço não se caracteriza como um espaço concreto, mas místico onde participantes podem "mover-se" e criar uma nova vida, explorando este mundo de informações ${ }^{3}$. Não é algo tangível, mas um espaço não físico onde seus integrantes têm a possibilidade de movimentar-se e criar-se explorando as oportunidades oferecidas por esse novo "mundo". Ele cria um mundo de simulação, onde às vezes a representação virtual se torna mais real do que o próprio mundo.
Este mundo virtual afeta, mesmo a contragosto de muitos, a sociedade atual. A tecnologia proporciona novas formas de sociabilização. A estas novas formas e possibilidades de sociabilização se denomina cibercultura.

O ciberespaço cria então, uma "feira de identidades" onde a identidade deste indivíduo é absorvida na rede criando novas "personalidades" onde o "eu" passa a ser sentido como fora do contexto real, especificado como um conflito psicológico ${ }^{4}$. Jovens no mundo todo criam novas formas de comunicação, denominada por Levy ${ }^{5}$, de cibercultura. Esta representaria toda uma nova geração, antes representada por movimentos sociais presenciais, agora representada via tecnologias. Assim como toda cultura e sociedade, também a considera excludente, por exigir aparelhagens que tem um valor agregado. Esta concepção de Levy ${ }^{5}$ tem se modificado ao longo dos anos, graças a inclusão digital e barateamento dos equipamentos de informática.

Lemos $^{6}$ relata que com a evolução da tecnologia novos termos foram incorporados a nossa cultura, entre eles, Cibercultura que é um termo que abrange os fenômenos relacionados ao ciberespaço, ou seja, fenômenos associados às formas de comunicação mediadas por computadores. Entretanto, o conjunto de objetos abrangidos pelo conceito é mais amplo. Levy ${ }^{5}$ afirma que para se entender a cibercultura, antes se passa pelo conceito de virtualidade.

Tem-se então um novo plano sociabilizador que pode servir como área de atuação para os profissionais de saúde pública, e não apenas como espaço para recrutamento de amostragem ou aplicação de instrumentos. Entender esse novo ambiente, suas peculiaridades se faz necessário para entender esse novo ser sócio-virtual que se apresente, que está interagindo e construindo um novo espaço que transcende o físico para algo virtualizado, sem essa dicotomia de real $\mathrm{x}$ virtual, onde ambos têm o mesmo significado para essas novas gerações. A Saúde Pública não pode ignorar a existência deste fenômeno e se faz preciso entender esse comportamento atual visando à saúde e bem estar deste novo ser digitalizado em pixels.

Com grande utilização e poder de acesso em nosso país, a Saúde Pública não poderia deixar de voltar seu olhar para esta ferramenta. Diversos estudos tiveram/têm como objeto esta rede mundial de computadores e sua interligação com o campo. Iturri ${ }^{7}$ e Cuenca et al. ${ }^{8}$ alertaram para a importância do uso das redes digitais nas instituições acadêmicas e pesquisas em saúde pública 
assim como Castro ${ }^{9}$ destaca o novo modelo de comunicação via rede, como um desafio para a academia moderna.

A vigilância epidemiológica pode se beneficiar com as ferramentas virtuais. Banaszek ${ }^{10}$ descreve como informações de fontes variadas, podem ser utilizadas nas identificações de possíveis focos de doenças. Conesa et al. ${ }^{11}$ afirmam que o software FluDetWeb é uma ferramenta muito útil para a saúde pública. Tilston et al. ${ }^{12}$ descrevem os benefícios de utilizar um sistema de vigilância digital. Freifeld et al. ${ }^{13}$ propõem um sistema de organização, filtragem, integração e visualização de todas as informações sobre doenças que são disponibilizadas na rede.

Além de fonte inesgotável de informações, a rede também pode ser utilizada como ferramenta para divulgação e disseminação de informações. Scotch et al. ${ }^{14}$ apontam para as dificuldades em se unificar as informações. Não deixam de ressaltar a importância de mais pesquisas, dado ao grande valor em potencial.

Vivancos et al. ${ }^{15}$ pesquisou tratamentos online para DST. Concluíram que estes produtos são de fácil acesso devido à facilidade que a Internet proporciona, assim como existe falta de informações sobre os riscos a que esses usuários podem estar se submetendo na compra destes produtos oferecidos na rede.

Gao et al. ${ }^{16}$ alertam para a necessidade de acompanhamento pela vigilância em saúde. Entretanto, afirmam que, para problemas mais complexos, soluções mais elaboradas são necessárias.

Chang et al. ${ }^{17}$ demonstram benefícios de utilização dessas tecnologias. Apresentam trabalho realizado com monitoramento, via Google Earth e mapeamento GIS, dos casos de dengue em países em desenvolvimento. Apontam essa ferramenta como adequada a regiões com pouco acesso às tecnologias e com resultados bastante satisfatórios, com baixo custo. Isso também é defendido por Lozano-Fuentes et al. ${ }^{18}$.

Na outra ponta da rede, a utilização de blogs é identificada como ferramenta importante em saúde pública. Esta seria uma ferramenta para expandir a experiência da sala de aula ${ }^{18-20}$. É uma fonte especial de consultas sobre sexualidade e informações sobre saúde, especialmente entre os adolescentes $^{21}$.

Chiou $^{22}$ pesquisou os efeitos do anonimato nos contatos de cibersexo realizados por adoles- centes em Taiwan. Facchini et al. ${ }^{23}$ pesquisaram sites voltados para adolescentes, sobre a temática sexualidade e concluem que há muita distorção de informações na Internet e reforço de comportamentos preconceituosos e sexistas, o que traz um alerta sobre o conteúdo a que os jovens têm acesso nos dias atuais.

$S_{\text {Silva }}{ }^{24}$ recentemente trabalhou os aspectos da sexualidade masculina online, focando nesses novos contextos de epidemia HIV/AIDS. Reforça a importância de considerarmos a Internet como campo de pesquisa, dados os aspectos socioculturais presentes. Nas relações entre pesquisas online e off-line, Ross et al. ${ }^{25}$ compararam resultados nas duas modalidades. Afirmam que a Internet é um campo favorável para pesquisas em sexualidade e saúde.

Hoje em dia, as pessoas encontram-se isoladas e distanciadas uma das outras, mesmo morando em regiões classificadas como de alta densidade demográfica. Às vezes é mais fácil saber o que está acontecendo em cidades e países distantes do que na sua própria vizinhança.

Com o advento da fibra ótica, computadores, conexões sem fio, $3 \mathrm{G}$ e outras tecnologias, se comunicar e buscar informações se tornou uma atividade cotidiana em qualquer lugar do mundo. A sociedade hoje é definida como a "sociedade da informação", onde estar conectado com o mundo e em contato direto com novas tecnologias e informações se faz necessário.

Com o uso em grande escala da Internet, era de se esperar que se tentasse reproduzir ao máximo a "sociedade real" na tentativa de atrair mais adeptos. A realidade criada por este novo meio de comunicação, que manifesta formas específicas de socialização, traz no seu bojo transformações de relações, de encontros, de possibilidades afetivas e cognitivas.

Com o passar dos anos, encontram-se cada vez mais utilidades para esta ferramenta, além de perceber que esse novo meio de comunicação tem gerado mudanças de comportamento, fazendo cada vez mais, parte do cotidiano das pessoas, sendo assim necessário o estudo desta interação homem-computador do ponto de vista sociopsicológico visando aos benefícios para a saúde pública.

$\mathrm{Na}$ atualidade, as mudanças ocorrem rapidamente, da mesma maneira que novos usuários adentram a rede, quase que sem controle. Essa nova apresentação de utilização de tecnologia tem o diferencial de corpo não presente. 


\section{Considerações finais}

As pesquisas e trabalhos em saúde pública e áreas transdisciplinares costumam encarar a virtualidade como um campo de aplicação de instrumentos, para terem acesso ou contato com os sujeitos, quando devemos pensar esta como um meio SOCIAL de interação, com características próprias e pertencente ao cotidiano dos sujeitos da sociedade moderna. Não se pode mais pensar na internet apenas como uma maneira de se realizar pesquisas ou divulgar instrumentos, mas sim com um campo social, virtualizado, onde existem novas normas e regras que atuam diretamente no comportamento desses nossos sujeitos modernizados e digitalizados.

Ha décadas pesquisadores se debruçam nas metodologias de pesquisa online, tentando recriar um ambiente mais próximo do real para realização das pesquisas. Pesquisas online não são apenas pesquisas reais que usam a rede como meio de propagação, mas sim pesquisas que entendem esse novo universo sociabilizador, com regras e normas próprias e que devem ser seguidas para evitarmos enviesamento.

Enquanto pesquisadores, precisamos entender o que é chamado de netiqueta, ou seja, normas de educação online, pois adentraremos um universo novo para novas pesquisas. Precisamos refletir como divulgaremos nossos instrumentos sem que invalidemos sua utilização, visto que as barreiras físicas não existem mais. Assim como entender o quesito anonimato e sua influência nos resultados bem como as novas formas de escrita e expressões que o virtual proporciona.

Existem novas formas de aplicação de instrumentos utilizando validação do termo de consentimento e esclarecido por Internet Protocol, que dão toda a segurança tanto ao usuário quanto aos pesquisadores. Diversos softwares estão sendo desenvolvidos para nos auxiliar, assim como os de estatística, no nosso trabalho de pesquisa- dores. Se faz necessário mais contato com as novas leituras desse mundo virtual social para que possamos inserir a saúde pública nesse novo contexto.

Devemos entender que novas gerações surgem e suas relações com o virtual também. Estudos sobre pesquisas com crianças e adolescentes no mundo virtual são realizadas e debatidas em grande escala. E as em Saúde pública? Estes ficarão fora das nossas pesquisas? Além desse ponto devemos pensar e repensar as questões de ensino em saúde pública, desde teleconferências ou tele saúde até novas formas de ensino e qualificação dos profissionais da nossa área. Não podemos deixar de citar que as pesquisas de mercado hoje utilizam os consumidores pelas redes sociais e novas formas de interação e precisamos dela para nossas atuações.

É necessário repensar as questões quantitativas, quando o universo e a amostra passam a ser incalculáveis ou até mesmo crescentes a cada minuto em progressão geométrica. Assim como entender como as metodologias de pesquisa nessa área precisam se adequar para este novo formato, sem perder sua essência. Em alguns poucos meses teremos mais e mais tecnologias que promovem a interação e a integração desses sujeitos e consequentemente dos pesquisadores. Estaremos nós preparados para esse novo desafio que antes era apenas um imaginário dos livros e filmes de ficção?

Existe um novo universo englobando o real, o chamado virtual, são dicotômicos, mas fundidos, criando novas formas de interação não geográfica, dinamizada e que não podem ser separadas nas pesquisas. Precisamos dessa integração da cibercultura com a saúde pública para que possamos acompanhar a sociedade e suas formas de interação e usar de melhor maneira este conhecimento e instrumentos para o desenvolvimento de políticas públicas, intervenções e pesquisas em saúde pública. 


\section{Referências}

1. Instituto Brasileiro de Geografia Estatística (IBGE) Pesquisa Nacional por Amostra de Domicílios. [dados na Internet]. [acessado 2010 jan 27]. Disponível em: http://www.ibge.gov.br/home/presidencia/ noticias/noticia_visualiza.php?id_noticia $=1517$

2. Le Breton D. Adeus ao Corpo: Antropologia e Sociedade. Campinas: Papirus Editora; 1999.

3. Carvalho PR. A psicologia social e a questão do virtual: pontuações temáticas na obra de Pierre Lévy. Psicologia 1999 [acessado 2004 ago 28];1(2): [cerca de 2 p.]. Disponível em: http://www2.uel.br/ccb/ psicologia/revista/resumovln23.htm

4. Parks MR, Floyd K. Making friends in cyberspace. Journal of Communication 1996; 46(1):80-97.

5. Levy P. Cibercultura. São Paulo: Ed. 34; 1999.

6. Lemos A. As estruturas antropológicas do ciberespaço. Salvador: texto produzido para os seminários do grupo Cyberpesquisa/Facom-UFBA, 1996. [página na Internet]. [acessado 2012 jan 20]. Disponível em: http://www.facom.ufba.br/pesq/cyber/lemos/ estrcyl.html

7. Iturri J. Ciberespaço e negociações de sentido: aspectos sociais da implementação de redes digitais de comunicação em instituições acadêmicas de saúde pública. Cad Saude Publica 1998; 14(4):803-810.

8. Cuenca AMB, Tanaka ACA. Influência da internet na comunidade acadêmico-científica da área de saúde pública. Rev Saude Publica 2005; 39(5):840-846.

9. Castro RCF. Impacto da Internet no fluxo da comunicação científica em saúde. Rev Saude Publica 2006; 40(N. Esp.):57-63.

10. Banaszek A. Tracking infectious diseases in cyberspace. CMAJ 2011; 183(7):E373-E374.

11. Conesa D, López-Quílez A, Martínez-Beneito MA, Miralles MT, Verdejo F. FluDetWeb: an interactive web-based system for the early detection of the onset of influenza epidemics. BMC Medical Informatics and Decision Making 2009; 9:36.

2. Tilston NL, Eames KTD, Paolotti D, Ealden T, Edmunds WJ. Internet-based surveillance of Influenza-like-illness in the UK during the $2009 \mathrm{H} 1 \mathrm{~N} 1$ influenza pandemic. BMC Public Health 2010; 10:650.

13. Freifeld CC, Mandl KD, Reis BY, Brownstein JS. HealthMap: global infectious disease monitoring through automated classification and visualization of Internet media reports. $J$ Am Med Inform Assoc 2008; 15(2):150-157.

14. Scotch M, Yip K Y, Cheung K H. Development of Grid-like Applications for Public Health Using Web 2.0 Mashup Techniques. J Am Med Inform Assoc 2008; 15(6):783-786.

15. Vivancos R, Schelenz S, Loke YK. Internet treatment of sexually transmitted infections - a public health hazard? BMC Public Health 2007; 7:333.

16. Gao S, Mioc D, Yi X, Anton F, Oldfield E, Coleman DJ. Towards Web-based representation and processing of health information. Int J Health Geogr 2009; 8:3.
17. Chang AY, Parrales ME, , Jimenez J, Sobieszczyk ME, Hammer SM, Copenhaver DJ, Kulkarni RP. Combining Google Earth and GIS mapping technologies in a dengue surveillance system for developing countries. Int J Health Geogr 2009; 8:49.

18. Lozano-Fuentes S, Elizondo-Quiroga D, Farfan-Ale JA, Loroño-Pino MA, Garcia-Rejon J, Gomez-Carro S, Lira-Zumbardo V, Najera-Vazquez R, Fernandez-Salas I, Calderon-Martinez J, Dominguez-Galera M, Mis-Avila P, Morris N, Coleman M, Moore CG, Beatya BJ, Eisena L. Use of Google EarthTM to strengthen public health capacity and facilitate management of vector-borne diseases in resource-poor environments. Bull World Health Organ 2008; 86:9.

19. Goldman RS, Cohen AP, Sheahan F. Using Seminar Blogs to Enhance Student Participation and Learning in Public Health School Classes. Am J Public Health 2008; 98(9):1658-1663.

20. Barilli ECVC, Ebecken NFF, Cunha GG. A tecnologia de realidade virtual como recurso para formação em saúde pública à distância: uma aplicação para a aprendizagem dos procedimentos antropométricos. Cien Saude Colet 2011; 16(Supl. 1):12471256.

21. Kanuga M, Rosenfeld WD. Adolescent sexuality and the internet: the good, the bad, and the URL. $J$ Pediatr Adolesc Gynecol 2004; 17(2):117-124.

22. Chiou WB. Adolescents' reply intent for sexual disclosure in cyberspace: gender differences and effects of anonymity and topic intimacy. Behav 2007; 10(5):725-728.

23. Facchini GB, Maia AB, Maia AF. Análise de aspectos relacionados à sexualidade em site para adolescentes. Interação em Psicologia 2004; 8(1):57-66.

24. Silva LAV. Cybersexuality and online research: some reflections about the concept of barebacking. Interface (Botucatu) 2010; 34(4):513-527.

25. Ross MW, Månsson SA, Daneback K, Cooper A, Tikkanen R. Biases in internet sexual health samples: Comparison of an internet sexuality survey and a national sexual health survey in Sweden. Soc Sci Med 2005; 61(1):245-252.

Artigo apresentado em 20/11/2012

Aprovado em 25/01/2013

Versão final apresentada em 04/02/2013 
